\title{
Island-wide diversity in single nucleotide polymorphisms of the Plasmodium vivax dihydrofolate reductase and dihydropteroate synthetase genes in Sri Lanka
}

\author{
Mette L Schousboe ${ }^{1}$, Rupika S Rajakaruna ${ }^{2}$, Ali Salanti \\ Hapuarachchige C Hapuarachchi ${ }^{3}$, Gawrie NL Galappaththy ${ }^{4}$, Ib C Bygbjerg1, \\ Priyanie H Amerasinghe ${ }^{5}$, Flemming Konradsen ${ }^{1}$ and Michael Alifrangis*1
}

\author{
Address: ${ }^{1}$ Centre for Medical Parasitology, Institute for International Health, Immunology and Microbiology, Øster Farimagsgade 5, 1014 \\ Copenhagen K, Denmark, ${ }^{2}$ Department of Zoology, University of Peradeniya, Peradeniya 20400, Sri Lanka, ${ }^{3}$ Department of Parasitology, Faculty \\ of Medicine, P.O. Box 6, Thallagolla Road, Ragama, Sri Lanka, ${ }^{4}$ Ministry of Health, Anti-Malarial Campaign Head Office, Colombo, Sri Lanka and \\ ${ }^{5}$ International Water Management Institute, Hyderabad, Andhra Pradesh, India \\ Email: Mette L Schousboe - Mette.Leth@cmp.dk; Rupika S Rajakaruna - Rupikar@pdn.ac.lk; Ali Salanti - Salanti@cmp.dk; \\ Hapuarachchige C Hapuarachchi - Chandith@lycos.com; Gawrie NL Galappaththy - Hapugalle@yahoo.co.uk; \\ Ib C Bygbjerg - I.Bygbjerg@pubhealth.ku.dk; Priyanie H Amerasinghe - P.Amerasinghe@cgiar.org; \\ Flemming Konradsen - F.Konradsen@pubhealth.ku.dk; Michael Alifrangis* - Alifrangis@cmp.dk \\ * Corresponding author
}

Published: 9 March 2007

Malaria Journal 2007, 6:28 doi:10.1186/1475-2875-6-28

This article is available from: http://www.malariajournal.com/content/6/1/28

(C) 2007 Schousboe et al; licensee BioMed Central Ltd.

This is an Open Access article distributed under the terms of the Creative Commons Attribution License (http://creativecommons.org/licenses/by/2.0), which permits unrestricted use, distribution, and reproduction in any medium, provided the original work is properly cited.

\begin{abstract}
Background: Single nucleotide polymorphisms (SNPs) in the Plasmodium vivax dihydrofolate reductase (Pfdhfr) and dihydropteroate synthetase (Pvdhps) genes cause parasite resistance to the antifolate drug combination, sulphadoxine/ pyrimethamine (SP). Monitoring these SNPs provide insights into the level of drug pressure caused by SP use and presumably other antifolate drugs. In Sri Lanka, chloroquine (CQ) with primaquine (PQ) and SP with PQ is used as first and second line treatment, respectively, against uncomplicated Plasmodium falciparum and/or $P$. vivax infections. CQ/PQ is still efficacious against $P$. vivax infections, thus SP is rarely used and it is assumed that the prevalence of SNPs related to $P$. vivax SP resistance is low. However, this has not been assessed in Sri Lanka as in most other parts of Asia. This study describes the prevalence and distribution of SNPs related to $P$. vivax SP resistance across Sri Lanka.
\end{abstract}

Subjects and methods: $P$. vivax-positive samples were collected from subjects presenting at government health facilities across nine of the major malaria endemic districts on the island. The samples were analysed for SNPs/haplotypes at codon 57, 58, 6I and II 17 of the Pvdhfr gene and 383, 553 and 585 of the Pvdhps gene by applying PCR followed by a hybridization step using sequence specific oligonucleotide probes (SSOPs) in an ELISA format.

Results: In the study period, the government of Sri Lanka recorded 2, 149 P. vivax cases from the nine districts out of which, 454 (21.1\%) blood samples were obtained. Pvdhfr haplotypes could be constructed for 373 of these. The FSTS wild-haplotype was represented in 257 samples $(68.9 \%)$, the double mutant LRTS haplotype was the most frequently observed mutant (24.4\%) while the triple mutation (LRTN) was only identified once. Except for two samples of the single mutated Pvdhps GAV haplotype, the remaining samples were wildtype. Geographical differences were apparent, notably a significantly higher frequency of mutant Pvdhfr haplotypes was observed in the Northern districts.

Conclusion: Since SP is rarely used in Sri Lanka, the high frequency and diversity of Pvdhfr mutations was unexpected indicating the emergence of drug resistant parasites despite a low level of SP drug pressure. 


\section{Background}

Plasmodium vivax is the most geographically widespread of the four Plasmodium species infective to humans found throughout South and Central America, Asia, the Middle East, and parts of Africa and infects an estimated 70-80 million people annually [1]. Chloroquine (CQ)-resistant Plasmodium falciparum, and to a lesser extent CQ resistant $P$. vivax, is almost as endemic as malaria itself and alternatives such as the drug combination sulphadoxine/ pyrimethamine (SP) have replaced CQ. Resistance to SP has recently emerged for $P$. falciparum, while for $P$. vivax it has been observed sporadically [2]. The molecular mechanisms involved in the development of SP resistance of the two species are most likely similar [3,4]. In P. falciparum, single nucleotide polymorphisms (SNPs) in codon (c) 51, c59 and c108 of the Pfdhfr gene and in c437 and c540 of Pfdhps gene provide pyrimethamine and sulphadoxine resistance, respectively and these SNPs combined result in high risk of SP treatment failure in vivo [5]. For $P$. vivax, the picture is more complex because pyrimethamine resistance possibly involve several SNPs [6]. However, some evidence support that resistance is mainly associated with mutations at c58 (S58R, occurring as two SNPs, either AGA $\left(R_{1}\right)$ or AGG $\left(R_{2}\right)$ ) and $c 117$ (S117N or S117T) with additional mutations at c57 (F57L-existing as three SNPs, CTC $\left(\mathrm{L}_{1}\right)$, TTG $\left(\mathrm{L}_{2}\right)$ and TTA $\left(\mathrm{L}_{3}\right)$ ) and $\mathrm{c} 61$ (T61M) in the Pvdhfr gene [3,4,6-8]. The quadruple mutant haplotype $(57 \mathrm{~L}+58 \mathrm{R}+61 \mathrm{M}+117 \mathrm{~T})$ has been shown to correlate with SP treatment failure in vivo [8] and increases $P$. vivax resistance to pyrimethamine by more than 500 times $[4,6]$.

Presumably, P. vivax sulphadoxine resistance is caused by SNPs in the Pvdhps gene. Based on homology models of both $P$. falciparum and P. vivax DHPS enzymes, Korsinczky et al. predicted that the P. vivax wildtype at c585 (V585) possibly cause some level of innate sulphadoxine resistance, while SNPs at c383 (A383G) and c553 (A553G) in Pvdhps most likely increase resistance levels [9]. Imwong et al. showed that only in regions with high SP usage, SNPs in both Pvdhfr and Pfdhps were observed and, furthermore, parasites harbouring multiple mutations in Pvdhfr and Pvdhps were cleared more slowly from the blood of patients following SP treatment [10]. Therefore, P. vivax $\mathrm{SP}$ resistance is most likely measurable by examining the frequency of SNPs in both the Pvdhfr and Pvdhps genes.

In Sri Lanka, CQ plus primaquine (PQ) and SP plus PQ are used as $1^{\text {st }}$ and $2^{\text {nd }}$-line treatment, respectively, against uncomplicated malaria infections, although CQ resistant $P$. falciparum infections have been reported since 1984 and $P$. falciparum SP resistance has been observed recently [11]. $P$. vivax resistance to either CQ or SP has not been recorded on the island. This study investigated the frequency of SNPs/haplotypes in the Pvdhfr (at c57, 58, 61 and 117) and Pvdhps (at c383, 553 and 585) genes in samples collected from nine districts with endemic $P$. vivax malaria in Sri Lanka over a 11 /2-year period. The detection of SNPs/haplotypes in Pvdhfr and Pvdhps was performed by applying a new simple enzyme-linked immunosorbent assay (ELISA) using sequence specific oligonucleotide probes (SSOPs) similar to the method detecting SNPs/haplotypes in P. falciparum $d h f r$, dhps and crt [12].

\section{Materials and methods}

The samples originated from individuals seeking treatment for malaria at government health facilities located in nine different malarious district across Sri Lanka. In Sri Lanka the great majority of individuals with perceived malaria seek treatment at government facilities [13]. Samples were collected by routine staff at the facilities trained by the Anti-Malaria Campaign (AMC) of Sri Lanka from September 2004 to March 2006, thereby including the traditionally malaria peak transmission seasons in January and one lower peak season around July. Finger prick blood from patients with single $P$. vivax or mixed $P$. vivax/ $P$. falciparum infections, diagnosed by microscopy were spotted on filter paper and sealed in individual zip-lock bags. DNA extraction was carried out by the chelex-100 method as described in [12].

As positive controls of the various Pvdhfr SNPs/haplotypes, $8 P$. vivax $d h f r$ allele samples, kindly provided by Carol Sibley (Dept. of Genome Sciences, University of Washington) were used [4]. These represent each of the ten most common c57, 58, 61 and 117 Pvdhfr SNPs/haplotypes. Furthermore, one positive control consisting of a 50 bp DNA fragment was designed mimicking a specific mutated Pvdhfr sequence, comprising the $\mathrm{L}_{3}$-mutation in c57 (TTA) and $\mathrm{R}_{2}$-mutation in c58 (AGG) $\left(\mathrm{PcL}_{3} \mathrm{R}_{2} \mathrm{~T}\right.$ in table 1 ) biotinylated at the 5 '-end by the supplier (MWG Biotech, Riskov, Denmark). Likewise for positive controls of Pvdhps 338G and 553G, 50 bp DNA fragments were designed mimicking these specific SNPs (Pc383G and Pc553G in Table 1).

The outer and nested Pvdhfr PCR protocols used are described in [14], with the exception that the reverse nested primer, $\mathrm{KH}-3 \mathrm{R}$ was biotinylated at the 5 '-end by the supplier (MWG Biotech, Riskov, Denmark). The outer Pvdhps PCR primers used (PvDHPS-D and PvDHPS-B) and protocols are described by [9]. The nested Pvdhps primers were designed; NL-1 (5'-GCGAGCGTGATTGACATC-3') and NR-1-(5'-GCTCATCAGTCTGCACTCC-3') where the reverse primer, NR-1, was biotinylated at the 5'end. The outer and nested Pvdhps PCR were performed as follows: denaturation at $94^{\circ} \mathrm{C}$ for $2 \mathrm{~min}$ followed by 40 cycles of $94^{\circ} \mathrm{C}$ for $30 \mathrm{sec}, 50^{\circ} \mathrm{C}$ for $30 \mathrm{sec}$ and $65^{\circ} \mathrm{C}$ for 1 $1 / 2 \mathrm{~min}$ and subsequently a $5 \mathrm{~min}$ extension step at $65^{\circ} \mathrm{C}$. 
Table I: The sequence specific oligonucleotide probes (SSOPs) targeting SNPs/haplotypes in c57, 58, 6 I and II 17 of the Pvdhfr gene and c383, 553 and 585 in the Pvdhps gene and artificial positive controls.

\begin{tabular}{|c|c|c|c|}
\hline SSOP & SSOP sequence $\dagger$ & Washing temperature $\S$ & Incubation time $\mathbb{\Upsilon}$ \\
\hline Pvdhfr & & $\left(C^{\circ}\right)$ & Min. \\
\hline 57/58/6I FST & AC TTC AGC TCG GTG ACG A & 60 & 12 \\
\hline $57 / 58 / 6 \mid \mathrm{FR}_{1} \mathrm{~T}$ & AC TTC AGA TCG GTG ACG A & 60 & 10 \\
\hline $57 / 58 / 61 \mathrm{FR}_{2} \mathrm{~T}$ & AC TTC AGG TCG GTG ACG A & 60 & 10 \\
\hline $57 / 58 / 61 L_{1} R_{2} M$ & ACCTC AGG TCG GTG ATG A & 62 & 10 \\
\hline $57 / 58 / 6 \mathrm{I} \mathrm{L} \mathrm{L}_{2} \mathrm{ST}$ & AC TTG AGC TCG GTG ACG A & 60 & 10 \\
\hline $57 / 58 / 6 \mid \mathrm{L}_{2} \mathrm{R}_{1} T$ & AC TTG AGA TCG GTGACG A & 64 & 10 \\
\hline $57 / 58 / 6 \mid \mathrm{L}_{3} \mathrm{R}_{2} T$ & AC TTA AGG TCG GTG ACG A & 62 & 10 \\
\hline $117 \mathrm{~s}$ & G AGA AGC AGC TGG GAG AG & 60 & 12 \\
\hline $117 \mathrm{~N}$ & G AGA AGC AAC TGG GAG AG & 60 & 10 \\
\hline $117 \mathrm{~T}$ & G AGA AGC ACC TGG GAG AG & 60 & 10 \\
\hline \multicolumn{4}{|l|}{ Pvdhps } \\
\hline $383 \mathrm{~A}$ & A TCG TCC GCC CCT TAT GT & 64 & 10 \\
\hline c383 G & A TCG TCC GGC CCT TAT GT & 64 & 10 \\
\hline c553A & TC GGC CTG GGG TTTGCC A & 64 & 10 \\
\hline c553G & TC GGC CTG GGG TTTGGC A & 64 & 10 \\
\hline$c 585 \mathrm{~V}$ & C TTT ATT GTC CAC TGC AT & 64 & 10 \\
\hline \multicolumn{4}{|c|}{ Positive controls* } \\
\hline Pvdhfr & Sequence & & \\
\hline $\mathrm{PcL}_{3} \mathrm{R}_{2} \mathrm{~T}$ & TCCGTCGATATGAAGTACTTAAGGTCGGTGACGACCTACGTGGATGAGTC & & \\
\hline \multicolumn{4}{|l|}{ Pvdhps } \\
\hline Pc383G & GATTGACATCGGGGGGGAATCGTCCGGCCCTTATGTGGTCCCCAATCCGA & & \\
\hline Pc553G & CTTTGATGTCGGCCTGGGGTTTGGCAAAAAGCACGACCAGTCTATTAAGC & & \\
\hline
\end{tabular}

† Sequences in bold indicate the codon in which the SNP occurs.

$\S$ Optimal TMAC-washing temperature.

II Optimal incubation time for TMAC-wash

* Artificial positive controls mimicking specific mutated Pvdhfr or Pvdhps sequences.

A SSOP-ELISA, similar to the method for SNP/haplotype analysis of $P$. falciparum $d h f r / d h p s$ was developed [12], however using pre-coated streptavidin plates (Nunc, Roskilde, Denmark). The 3'-end digoxigenin-conjugated SSOPs designed to target the most common Pvdhfr and Pvdhps SNP/haplotypes including the time and temperatures in the two rounds of high stringency washing with tetra-methyl-ammonium chloride (TMAC) is given in table 1. Scoring of ELISA data were performed as described elsewhere [12]. To verify the results, Restriction Fragment Length Polymorphism (RFLP) was performed on a subset of the samples using enzymes and methods described by [15]. Polymorphisms in c383 of the Pvdhps gene were identified by digestion with the restriction enzyme HaeIII (New England Biolabs, Medinova, Glostrup, Denmark).

Sequencing was performed on a subset of samples to clarify some of the Pvdhfr and Pvdhps haplotypes; PCR products with A-overhang were cloned into the TOPO TA vector according to manufacturing procedures (Invitrogen), and plasmids were prepared using MiniPrep spin columns (Omega Biotech). Sequencing was done on an ABI Prism 377 (Perkin-Elmer) using the Big Dye terminator reaction mix (Perkin-Elmer).
Ethical clearance for this project was granted by the Committee on Research and Ethical Review at the Faculty of Medicine, Peradeniya, Kandy and verbal consent was obtained from participants, parents and/or guardians.

\section{Results}

In the study period, AMC recorded a total of 2717 P. vivax cases in the country, out of which, 2,149 cases came from the nine districts included in this study (79.1\%). 454 (21.1\%) blood samples from these districts, representing a large range of catchments efficiencies from 7.7\% (Monaragala) to $67.5 \%$ (Polonnaruwa) were examined. The samples were analysed for SNPs/haplotypes at position c57, 58, 61 and 117 of the Pvdhfr gene and c383, 553 and 585 (only detection of the wildtype V585) of the Pvdhps gene using an array of SSOPs. Samples either repeatedly PCR negative or negative in one or more of the Pvdhfr or Pvdhps codons were omitted from the analysis.

For Pvdhfr, haplotypes could be constructed for 373 samples ( $84.6 \%$ ) including 25 samples with mixed haplotype infections, but where a major haplotype could be deduced (Figure 1). The Pvdhfr FSTS wild haplotype was represented in 257 samples (68.9\%) while the remaining sam- 
ples comprised of five different mutant haplotypes at c57, 58 and 117 only: as $\mathrm{F}^{2} 7 \mathrm{~L}_{3} \quad$ (TTC $\rightarrow$ TTA), $\quad$ S58R (AGC $\rightarrow$ AGG) and S117N. The double mutant LRTS haplotype was the most frequently mutated haplotype, observed in the districts Anuradhapura (frequency (f): $0.05, \mathrm{n}=10$ ), Trincomalee (f: $0.52, \mathrm{n}=29$ ), Pollonaruwa (f: $0.18, \mathrm{n}=9$ ), Batticaloa (f: $0.43, \mathrm{n}=6$ ), Kurunegala (f: $0.71, \mathrm{n}=34$ ) and Ampara (f: $0.43, \mathrm{n}=3$ ). The $117 \mathrm{~N}$ mutation was less frequently observed (in total: $\mathrm{f:} 0.06, \mathrm{n}=21$ ), as either the single mutated haplotype FSTN (only in Polonnaruwa and Anuradhapura), the double mutated haplotype, FRTN (only in the Northern districts of Mannar, Vavuniya and Trincomalee). The triple mutation (LRTN) was only identified once in the district Pollonaruwa and as a mixture of LRTN/LRTS with the LRTN in majority. A significantly higher frequency of mutant haplotypes (FSTN, FRTN and LRTS) was observed in the Northern districts (Mannar, Trincomalee and Vavuniya) compared to the other districts $\left(\chi^{2}=36.3, \mathrm{P} \leq 0.001\right)$. There was a general tendency for an increase in the frequency of mutant haplotypes late in the study period. However, due to a skewed temporal collection of samples from the various districts the differences was not analysed further.

A subset of samples analysed by sequencing (mainly to confirm the $c 57 \mathrm{~L}_{3}$ ) and by digestion of $\mathrm{c} 58$ and 117 in the Pvdhfr gene by RFLP confirmed the data obtained be the Pvdhfr SSOP-ELISA.

The Pvdhps haplotypes could be constructed for 368 of the $373 \mathrm{Pvdhfr}$ positive samples (98.7\%). Wildtype haplotypes at c383, 553 and 585 (AAV) was seen in 366 of these samples, while two samples from Trincomalee were of the single mutated GAV haplotype. These were confirmed by sequencing. Both samples expressed the double mutated FRTN haplotype in Pvdhfr.

\section{Discussion}

The present descriptive study analysed sulphadoxine/ pyrimethamine (SP) resistance-related SNPs in the $P$. vivax dhfr and Pvdhps genes in samples originating from nine districts in Sri Lanka, a country were both CQ and SP (in combination with primaquine) is still regarded as efficient treatment against uncomplicated $P$. falciparum and/ or $P$. vivax infections. The analysis identified six different haplotypes of $P v d h f r$ while for $P v d h p s$, only wildtypes were identified except for two cases.

The double mutant haplotype LRTS (F57L, S58R, T61,117S) was the most frequent mutant haplotype and not as expected as a combination of S117N and S58R (FRTN) as observed previously $[6,8,16]$ and in a recent study from India [17]. The P. vivax triple mutant haplotype LRTN, previously found in Thailand and shown to be associated with reduced ability of patients to decrease par- asites ratios [15] was only found once and the quadruple LRMT mutant haplotype causing a high risk of SP treatment failures [8] was not detected, thus indicating that SP (with primaquine) is still efficient against $P$. vivax infections in Sri Lanka. Nevertheless, it is surprising that almost one third of the tested $P$. vivax infections were mutated in the Pvdhfr gene, despite that, officially, SP is only used as second-line drug against CQ treatment failures of $P$. falciparum. A recent study investigating the availability of SP in privately-owned drug vendor shops in Sri Lanka found that SP was virtually absent from the shops [18], thus the specific drug pressure is unlikely to be caused by unauthorized use. More plausible, the mutations are not only an indication of emerging pyrimethamine resistance, but instead reflect the overall antifolate pressure in Sri Lanka. Presently, antifolates such as dapsone, co-trimoxazole and trimethoprim are for instance used against urinary tract infections and chronic bronchitis on the island. Alternatively, similar to development of $P$. falciparum resistance to pyrimethamine in vivo, $P$. vivax populations are occasionally exposed to sub-therapeutic levels of pyrimethamine when re-infecting recently SP-treated patients thereby providing optimal conditions for the emergence of SP tolerant $P$. vivax parasites [19]. Thus, even low drug pressure may facilitate the emergence of drug tolerant/resistant parasites and this may particularly be the case for $P$. vivax that to a larger extend than P. falciparum possibly can persist in the host unnoticed.

The frequency of Pvdhfr mutant haplotypes was significantly higher in the most Northern regions (Mannar, Vavuniya and Trincomalee) than the rest of the districts examined. This might be indirectly caused by the civil unrest resulting in a shortage of trained medical personnel, non-accurate malaria diagnosis and an underestimation of malaria infections mainly in the Northern part of Sri Lanka [20]. Furthermore, the FRTN haplotype was only observed in the Northern districts and it may be speculated that human migration between Southern India and the Northern part of Sri Lanka has introduced this particular haplotype from the Southern district of Chennai where it is highly prevalent [17]. The limited number of samples received from some districts, e.g. Monaragala, Ampara, Batticaloa, Mannar and Vavuniya, limits interpretation.

The high frequency of mutant haplotypes related to pyrimethamine resistance is worrying because it indicates that drug tolerant/resistant $P$. vivax parasites have evolved despite a low level of SP drug pressure, possibly attributed to the use of other antifolate drugs. It is not known whether these mutant $P$. vivax haplotypes do exhibit SP resistance in vivo. 


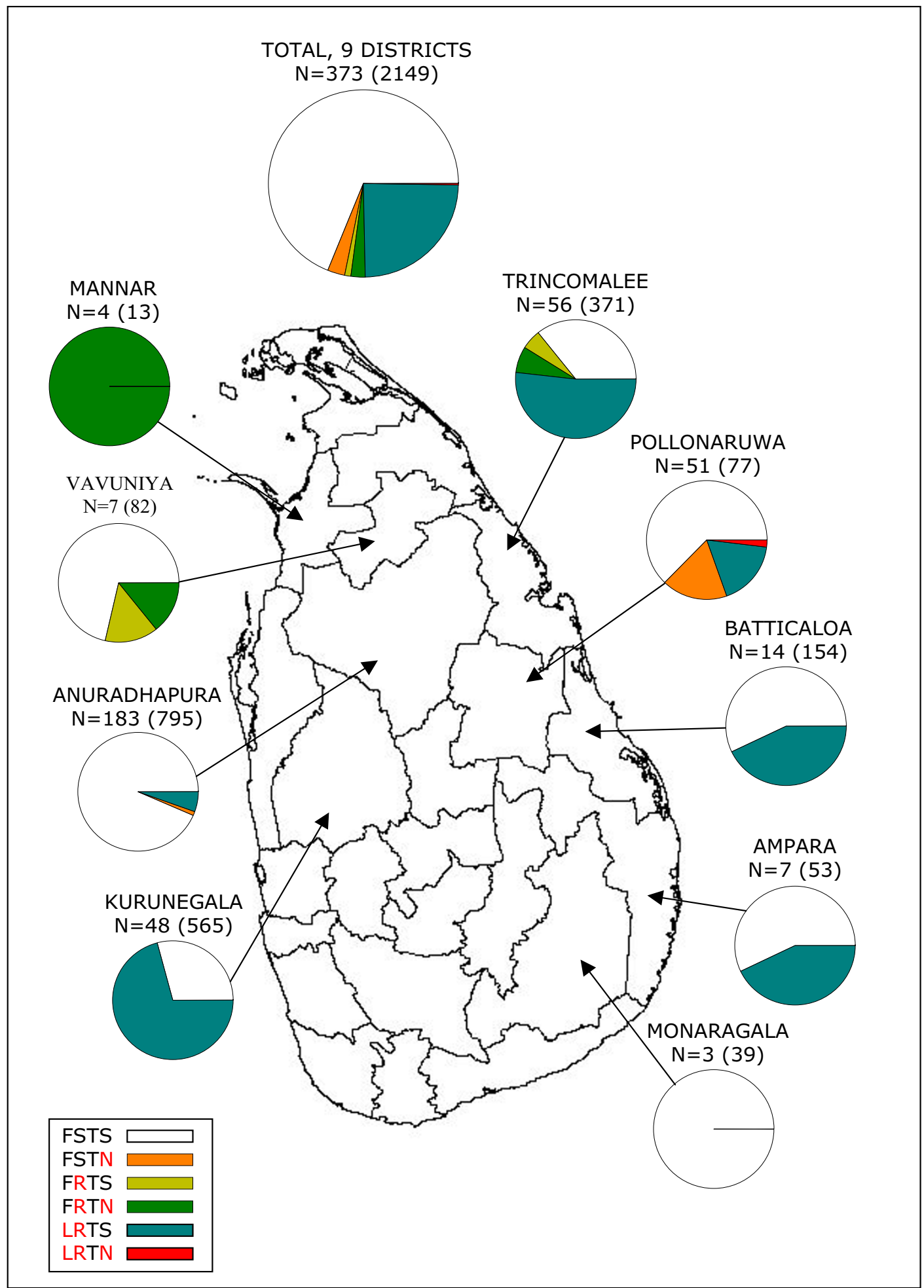

Figure I

The distribution of Plasmodium vivax dhfr haplotypes at c57 (F->L), 58 (S->R), 6 I (T) and I I 7 (S->N) in samples collected from $\boldsymbol{P}$. vivax positive patients in $\mathbf{9}$ districts of Sri Lanka. The number in brackets is the actual number of $P$. vivax positive cases reported by Anti Malaria Campaign of Sri Lanka in the study period September 2004-March 2006. In red: the wild type to mutant type change in amino acids 


\section{Authors' contributions}

RSR, GNLG, ICB, PHA, FK and MA designed the study, MLS carried out the molecular studies, MA, FK and MLS analysed the data, AS performed the sequencing experiments. HCH provided valuable inputs in the drafting of the manuscript. All authors read and approved the final manuscript.

\section{Acknowledgements}

The Regional Medical Officers and the technical personnel are thanked for supplying the filter paper blood samples and laboratory technician Ulla Abildrup are thanked for excellent technical assistance. Carol Sibley are thanked for kindly providing the $P$. vivax dhfr samples.

\section{References}

I. Mendis K, Sina BJ, Marchesini P, Carter R: The neglected burden of Plasmodium vivax malaria. Am J Trop Med Hyg 200I, 64:97-106.

2. Pukrittayakamee S, Imwong M, Looareesuwan S, White NJ: Therapeutic responses to antimalarial and antibacterial drugs in vivax malaria. Acta Trop 2004, 89:35I-356.

3. Eldin P, Basco LK, Tahar R, Ouatas T, Mazabraud A: Analysis of the Plasmodium vivax dihydrofolate reductase-thymidylate synthase gene sequence. Gene 1998, 21 I: I77-185.

4. Hastings MD, Porter KM, Maguire JD, Susanti I, Kania W, Bangs MJ, Hopkins SC, Baird JK: Dihydrofolate Reductase Mutations in Plasmodium vivax from Indonesia and Therapeutic Response to Sulfadoxine Plus Pyrimethamine. J Infect Dis 2004, I 89:744-750.

5. Kublin JG, Dzinjalamala FK, Kamwendo DD, Malkin EM, Cortese JF, Martino LM, Mukadam RA, Rogerson SJ, Lescano AG, Molyneux ME, Winstanley PA, Chimpeni P, Taylor TE, Plowe CV: Molecular markers for failure of sulfadoxine-pyrimethamine and chlorproguanil-dapsone treatment of Plasmodium falciparum malaria. J Infect Dis 2002, 185:380-388.

6. Hastings MD, Maguire JD, Bangs MJ, Zimmerman PA, Reeder JC, Baird $\mathrm{JK}$, Sibley $\mathrm{CH}$ : Novel Plasmodium vivax dhfr alleles from the Indonesian Archipelago and Papua New Guinea: association with pyrimethamine resistance determined by a Saccharomyces cerevisiae expression system. Antimicrob Agents Chemother 2005, 49:733-740.

7. Imwong M, Pukrittayakamee S, Renia L, Letourneur F, Charlieu JP, Leartsakulpanich U, Looareesuwan S, White NJ, Snounou G: Novel point mutations in the dihydrofolate reductase gene of Plasmodium vivax: evidence for sequential selection by drug pressure. Antimicrob Agents Chemother 2003, 47:|5|4-|52|.

8. Tjitra E, Baker J, Suprianto S, Cheng Q, Anstey NM: Therapeutic efficacies of artesunate-sulfadoxine-pyrimethamine and chloroquine-sulfadoxine-pyrimethamine in vivax malaria pilot studies: relationship to Plasmodium vivax dhfr mutations. Antimicrob Agents Chemother 2002, 46:3947-3953.

9. Korsinczky M, Fischer K, Chen N, Baker J, Rieckmann K, Cheng Q: Sulfadoxine resistance in Plasmodium vivax is associated with a specific amino acid in dihydropteroate synthase at the putative sulfadoxine-binding site. Antimicrob Agents Chemother 2004, 48:221 4-2222.

10. Imwong M, Pukrittayakamee S, Cheng Q, Moore C, Looareesuwan S, Snounou G, White NJ, Day NP: Limited polymorphism in the dihydropteroate synthetase gene (dhps) of Plasmodium vivax isolates from Thailand. Antimicrob Agents Chemother 2005, 49:4393-4395.

II. Hapuarachchi HC, Dayanath MY, Bandara KB, Abeysundara S, Abeyewickreme W, de Silva NR, Hunt SY, Sibley CH: Point mutations in the dihydrofolate reductase and dihydropteroate synthase genes of Plasmodium falciparum and resistance to sulfadoxine-pyrimethamine in Sri Lanka. Am J Trop Med Hyg 2006, 74:198-204.

12. Alifrangis M, Enosse S, Pearce R, Drakeley C, Roper C, Khalil IF, Nkya WM, Ronn AM, Theander TG, Bygbjerg IC: A simple highthroughput method to detect Plasmodium falciparum single nucleotide polymorphisms in the dihydrofolate reduct- ase, dihydropteroate synthase, and $P$. falciparum cloroquine resistance transporter genes using ploymerase chain reaction and enzyme linked immunosorbent assay-based technology. Am J Trop Med Hyg 2005, 72: I55- 162.

13. Konradsen F, Van der HW, Amerasinghe PH, Amerasinghe FP, Fonseka KT: Household responses to malaria and their costs: a study from rural Sri Lanka. Trans R Soc Trop Med Hyg 1997, 91:127-130.

14. de Pecoulas PE, Tahar R, Yi P, Thai KH, Basco LK: Genetic variation of the dihydrofolate reductase gene in Plasmodium vivax in Snoul, northeastern Cambodia. Acta Trop 2004, 92: I-6.

15. Imwong M, Pukrittakayamee S, Looareesuwan S, Pasvol G, Poirreiz J, White NJ, Snounou G: Association of genetic mutations in Plasmodium vivax dhfr with resistance to sulfadoxine-pyrimethamine: geographical and clinical correlates. Antimicrob Agents Chemother 200I, 45:3I22-3I 27.

16. de Pecoulas PE, Tahar R, Ouatas T, Mazabraud A, Basco LK: Sequence variations in the Plasmodium vivax dihydrofolate reductase-thymidylate synthase gene and their relationship with pyrimethamine resistance. Mol Biochem Parasitol 1998, 92:265-273.

17. Kaur S, Prajapati SK, Kalyanaraman K, Mohmmed A, Joshi H, Chauhan VS: Plasmodium vivax dihydrofolate reductase point mutations from the Indian subcontinent. Acta Trop 2006, 97: I 74- 80.

18. Rajakaruna RS, Weerasinghe M, Alifrangis M, Amerasinghe PH, Konradsen $F$ : The role of private drug vendors as malaria treatment providers in selected malaria endemic areas of Sri Lanka. J Vector Borne Dis 2006, 43:58-65.

19. Hastings IM, Watkins WM: Tolerance is the key to understanding antimalarial drug resistance. Trends Parasitol 2006, 22:7I-77.

20. Briet OJ, Gunawardena DM, Van der HW, Amerasinghe FP: Sri Lanka malaria maps. Malar J 2003, 2:22.
Publish with Bio Med Central and every scientist can read your work free of charge

"BioMed Central will be the most significant development for disseminating the results of biomedical research in our lifetime. "

Sir Paul Nurse, Cancer Research UK

Your research papers will be:

- available free of charge to the entire biomedical community

- peer reviewed and published immediately upon acceptance

- cited in PubMed and archived on PubMed Central

- yours - you keep the copyright

Submit your manuscript here:

http://www.biomedcentral.com/info/publishing_adv.asp 